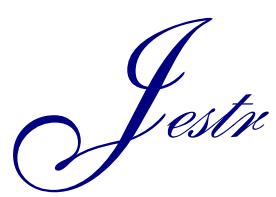

Journal of Engineering Science and Technology Review 4 (3) (2011) 297 -300 Special Issue on Econophysics

Research Article

\title{
Predicting the CDS return index
}

\author{
M. P. Hanias ${ }^{1, *}$, L. Magafas ${ }^{2}$ and A. Ozun ${ }^{3}$ \\ ${ }^{I}$ Dep. of Electronics, Computers, Telecommunications, and Control, Faculty of Physics, \\ University of Athens, Panepistimiopolis, Athens 15784, Greece \\ ${ }^{2}$ Dep.of Electrical Engineering, Kavala Institute of Technology, St. Loukas 65404 Kavala, Hellas. \\ ${ }^{3}$ IS Bank of Turkey, Market Risk Management, 34330, Istanbul, Turkey
}

\begin{abstract}
This paper sets out to apply concepts of non linear dynamics theory nai neural networks to the prediction of CDS index using Greek, Turkey, Russia, Brazil and China data. The research employs the method of false near neighbors in the time series analysis in order to estimate the minimum membedding dimensions of the corresponding strange attractor. To achieve out of the sample multistep ahead prediction, a neural net is constructed which architectures based on strange attractors topological properties
\end{abstract}

Keywords: CDS index, False Neighbors, Neural networks, Greece, Turkey, Russia, Brazil ,China

\section{Introduction}

Non linear dynamics [1] in combination with neural networks had applied in a wide variety of fields, e.g. physics, engineering, ecology and economics . The economist interest is focus on the ability of forecasting an economic time series as the CDS index. In this work we have applied non linear time series analysis in daily closing values of Greece,Turkey,Russia,Brazil and China CDS index. The daily values of historical data are received from Bloomberg Plc. We cover time period from 2-01-2008 until 23-06-2010. We have applied the method of false near neighbours $[2,3]$ to evaluate the minimum embedding dimension of each the system. In a second stage using the neural network [4] we achieved an out of sample multi step time series prediction.

\section{Time Series}

The CDS return log index is presented as a signal $\mathrm{x}=\mathrm{x}(\mathrm{t})$ where $\mathrm{x}=\log (\mathrm{CDS})$, as it shown at Figure 1 for Greece,Turkey,Russia,Brazil and China.. It covers data from $2-1-2008$ to $23-06 / 2010$. The sampling rate is $\Delta \mathrm{t}=1$ day and the number of data are $\mathrm{N}=645$.

\section{State Space Reconstruction}

From our data we construct a vector $\vec{X}_{i}, \mathrm{i}=1$ to $\mathrm{N}$, in the $\mathrm{m}$ dimensional phase space given by the following relation

\footnotetext{
*E-mail address: mhanias@gmail.com

ISSN: 1791-2377 @ 2011 Kavala Institute of Technology. All rights reserved.
}

$[5,6]$ :

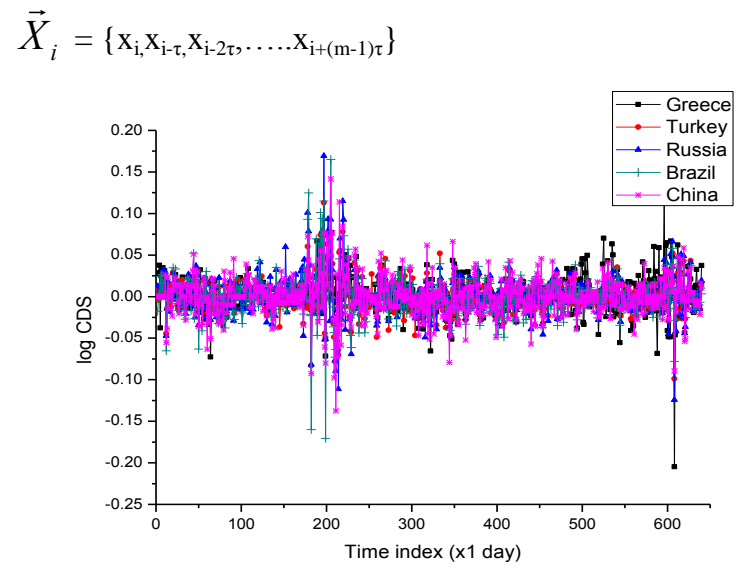

Fig. 1 Time series of $\log$ CDS

This vector, represents a point to the $\mathrm{m}$ dimensional phase space in which the attractor is embedded each time, where $\tau$ is the time delay $\tau=\mathrm{i} \Delta \mathrm{t}$. The element $\mathrm{x}_{\mathrm{i}}$ represents a value of the examined scalar time series in time, corresponding to the $i$-th component of the time series. Use of this method reduces phase space reconstruction to the problem of proper determining suitable values of $m$ and $\tau$. The choice of these values is not always simple, especially when we do not have any additional information about the original system and the only source of data is a simple sequence of scalar values, acquired from the original system. The dimension, where a time delay reconstruction of the phase space provides a necessary number of coordinates to unfold the dynamics from overflaps on itself caused by projection is called embedding dimension $m$. 


\subsection{Time delay $\tau$}

Using the average mutual information we can obtaining $\tau$, less associated with linear point of view, and thus more suitable for dealing with nonlinear problems. The average mutual information, which may be expressed by the following formula $[7,8]$

$$
I(\tau)=\sum_{x_{i}, x_{i+\tau}} P\left(x_{i}, x_{i+\tau}\right) \log _{2}\left(\frac{P\left(x_{i}, x_{i+\tau}\right)}{P\left(x_{i}\right) P\left(x_{i+\tau}\right)}\right)
$$

where $P\left(x_{i}\right)$ represents probability of value $x_{i}$ and $P\left(x_{i}, x_{i+\tau}\right)$ is joint probability. In general, $I(\tau)$ expresses the amount of information (in bits), which may be extracted from the value in time $x_{i}$ about the value in time $x_{i+\tau}$. As $\tau$, suitable for the phase space reconstruction, is the first minimum of $\mathrm{I}(\mathrm{t})$. Also the absolute minimum of $I(\tau)$ is used for decorrelation time. The results are presented at Table. 1

Table .1 Absolute minimum of Average mutual information

\begin{tabular}{l|c|l}
\hline Countries & First minimum of I(t) & $\begin{array}{l}\text { Absolute minimum of } \\
\mathrm{I}(\tau)\end{array}$ \\
\hline Greece & 3 & 45 \\
\hline Turkey & 1 & 48 \\
\hline Russia & 3 & 35 \\
\hline Brazil & 2 & 39 \\
\hline China & 1 & 39
\end{tabular}

\subsection{Embedding dimension $m$}

Embedding theorems show that there is always a dimension $\mathrm{m}$ for which the geometric object formed by $\vec{X}_{i}$ is equivalent to the original trajectory. A technique to estimate the optimal delay dimension $\mathrm{m}$ is by looking for false neighbors in phase space, [9-11]. More specifically, the method is based on a fact that when embedding dimension is too low, the trajectory in the phase space will cross itself. If we are able to detect these crossings, we may decide whether the used $\mathrm{m}$ is large enough for correct reconstruction of the original phase space (i.e. when no intersections occur) or not. When intersections are present for a given $\mathrm{m}$, the embedding dimension is too low and we have to increase it at least. Then, we test the eventual presence of self-crossings again until find a characteristic embedding dimension at which the trajectory in the phase space will not cross itself. A noise free time series, theoretically, would not have any false neighbors for an embedding dimension $m$ larger than a minimum value. This minimum embedding dimension is as we said before the embedding dimension at which the trajectory in the phase space will not cross itself. If we increase more the embedding dimension and repeat the procedure, we would not have any false neighbors. We implement the false nearest-neighbor algorithm in log CDS data with delay times according the values of first minimum of $I(\tau)$ from Table -1 , decorrealation time the values of absolute minimum of $\mathrm{I}(\mathrm{t})$ from Table -1 ,number of neighbors equal to 20 , and calculating the distance between two points, using the Euclidian norm, in order to judge whether these points are false neighbors at upper dimensions or not.

The practical realization of the described method is based on testing of the neighboring points in m-dimensional phase space. Typically, we take a certain amount of points in the phase space and find the nearest neighbor to each of them. Then we compute distances for all these pairs and also their distances in $(\mathrm{m}+1)$-dimensional phase space. The rate of these distances is given by

$P=\frac{\left\|\vec{X}_{i}(m+1)-\vec{X}_{n(i)}(m+1)\right\|}{\left\|\vec{X}_{i}(m)-\vec{X}_{n(i)}(m)\right\|}$

where $\vec{X}_{i}(m)$ represents the reconstructed vector as described in eq. (1), belonging to the $\mathrm{i}$-th point in the $\mathrm{m}$ dimensional phase space and index $n(i)$ denotes the nearest neighbor to the $\mathrm{i}$-th point. If $\mathrm{P}$ is greater than some value Pmax, we call this pair of points false nearest neighbors (i.e. neighbors, which arise from trajectory self-intersection and not from the closeness in the original phase space). In the ideal case, the value of $\mathrm{m}$ is found when the number of false neighbors falls to zero. For this purpose, we compute the rate of false nearest neighbours in the reconstructed phase space using the formula

$$
\left|X_{i+m \tau}-X_{n(i)+m \tau}\right| \geq R_{A}
$$

where $R_{A}$ is the radius of the attractor,

$$
R_{A}=\frac{1}{N} \sum_{i=1}^{N}\left|X_{i}-\bar{X}\right|
$$

and

$$
\bar{X}=\frac{1}{N} \sum_{i=1}^{N} X_{i}
$$

is the average value of time series.

When the following criterion

$$
P \geq P_{\max }
$$

is satisfied, then it can be used to distinguish between true and false neighbours. The dimension $\mathrm{m}$ will be found when the false nearest neighbors percentage falls below some limit, typically set to $1 \%$, [12], and, thus, by choosing $P \max =10$ and using Matlab code we finally calculate the quantity $\mathrm{P}$. The so obtained results are shown in Fig. 2 indicating that the application of the FNN method yields a minimum embedding dimension $m$ equal to $5,4,5,4,5$ for Greece, Turkey,Russia,Brazil and China

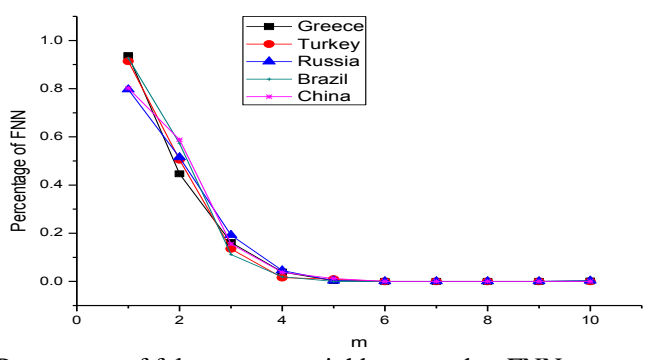

Fig.2 Percentage of false nearest neighbors number FNN vs. $m$ 


\section{Time series prediction}

The neural network model is especially useful if the time series show non-linear patterns. In emerging markets, the returns on financial instruments have non-linear behaviors. Additionally, the entropy in the emerging markets are required a special examination by non-linear models. Thus, to examine the performance of the neural networks models efficiently, emerging markets data is used. For this purpose we construct a backpropagation network [4, 13, 14] that consists of 1 input layer 2 middle or hidden layers and 1 output layer. The input layer has number of neurons equal to the value of absolute minimum of $\mathrm{I}(\mathrm{t})$, the $1^{\text {st }}$ hidden layer has the same number of neurons as the input layer the $2^{\text {nd }}$ middle layer has $4 \mathrm{~m}$ neurons as a rule of thumb. We choose the input and $1^{\text {st }}$ hidden layer to have this number of inputs to avoid temporal correlation, and because the attractor is embedded at a m phase space the last hidden layer has $4 \mathrm{~m}$ neurons . As an example for Greece, beginning with the first set of inputs $\mathrm{x} 1, \mathrm{x} 2, \mathrm{x} 3 \ldots \mathrm{x} 45$ the output is the $\mathrm{x} 46$. Then with an iterative process we attempt to predict the next 20 values until x66. We repeat the process for all training sets. We train the network with a training set of 600 exemplars using the $70 \%$ of each data set. The learning rate $\beta=0.05$ and the momentum $\alpha=0.5[4]$. Each network was training for 100000 epochs After 100000 epochs the training mean square error was ,MSE $=10^{-6}$. We applied the same procedure for CDS index for the other countries too. In figure 3 in sample actual and predicted values during the learning process are presented. While in figure 4 out of sample actual and predicted values are presented using the multistep iterative prediction process
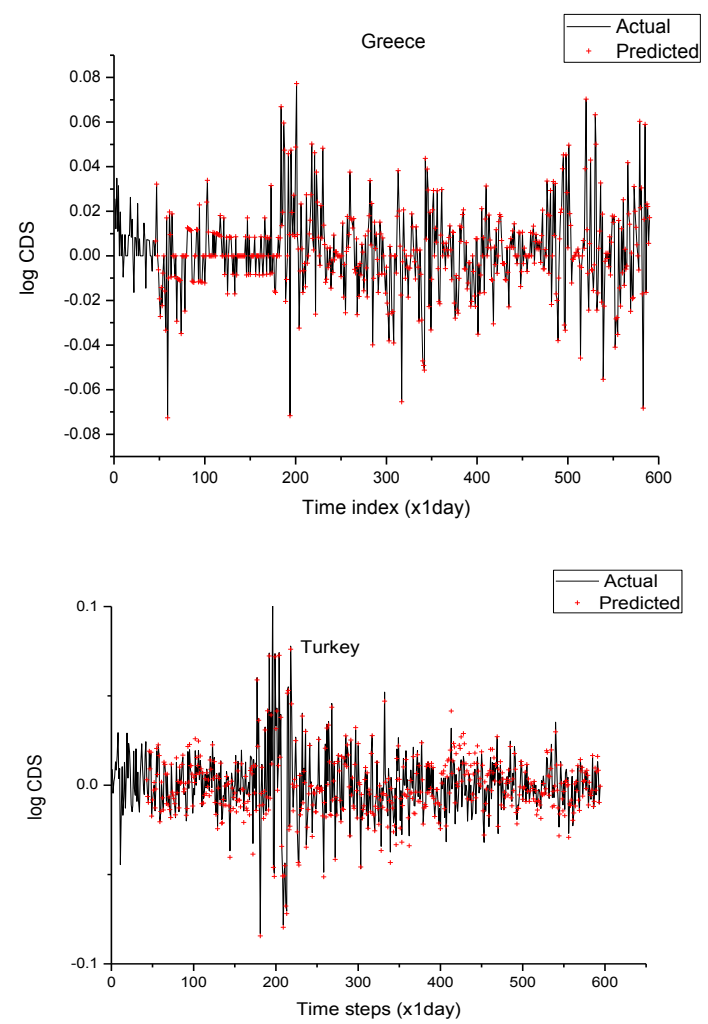
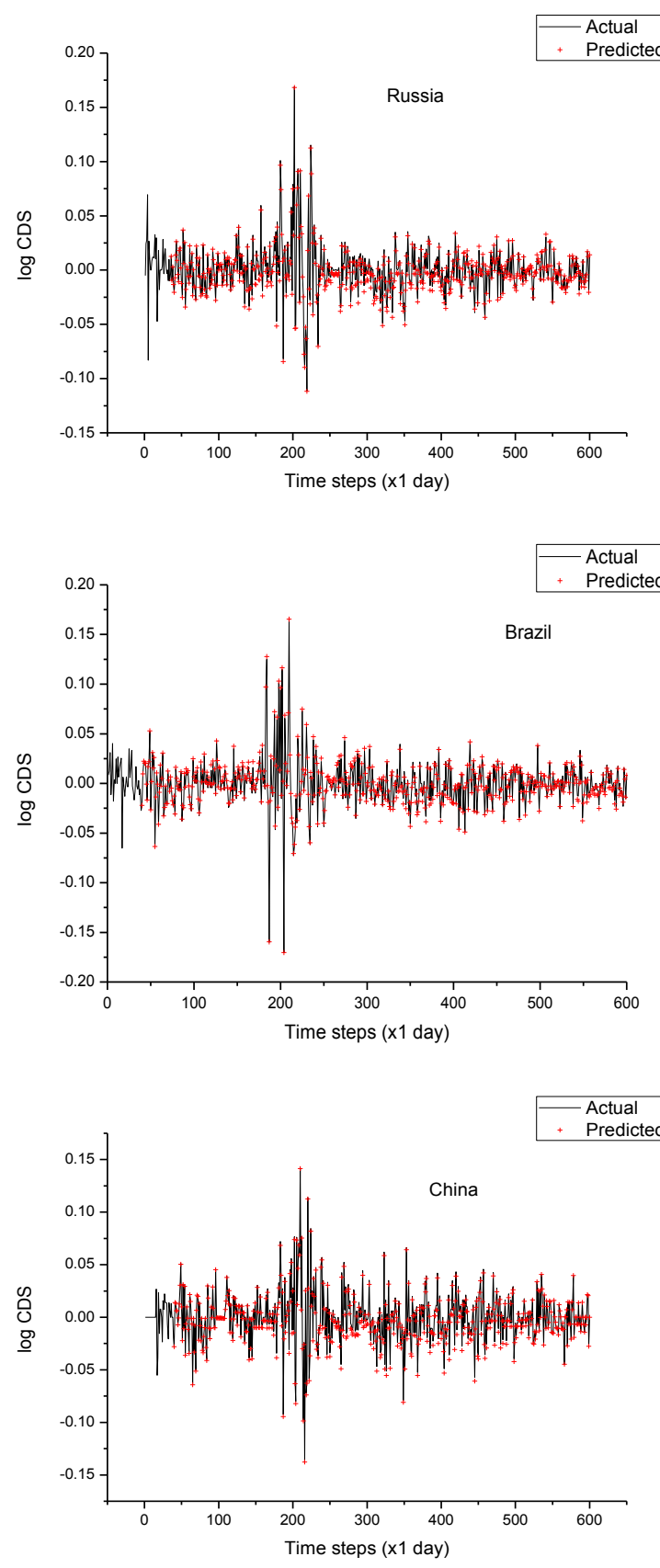

Fig.3 In sample actual and predicted values during the learning process

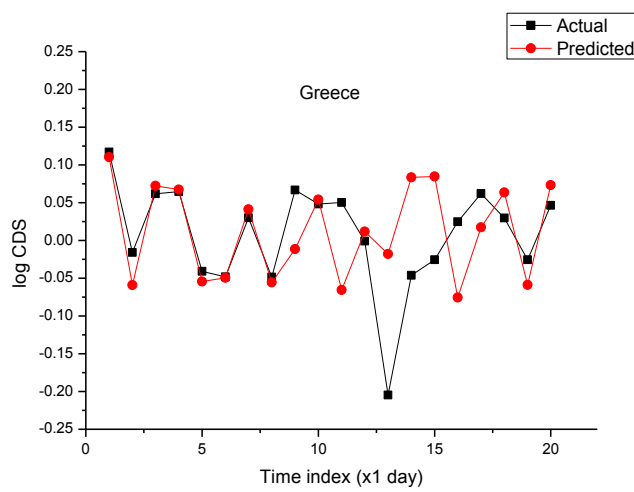



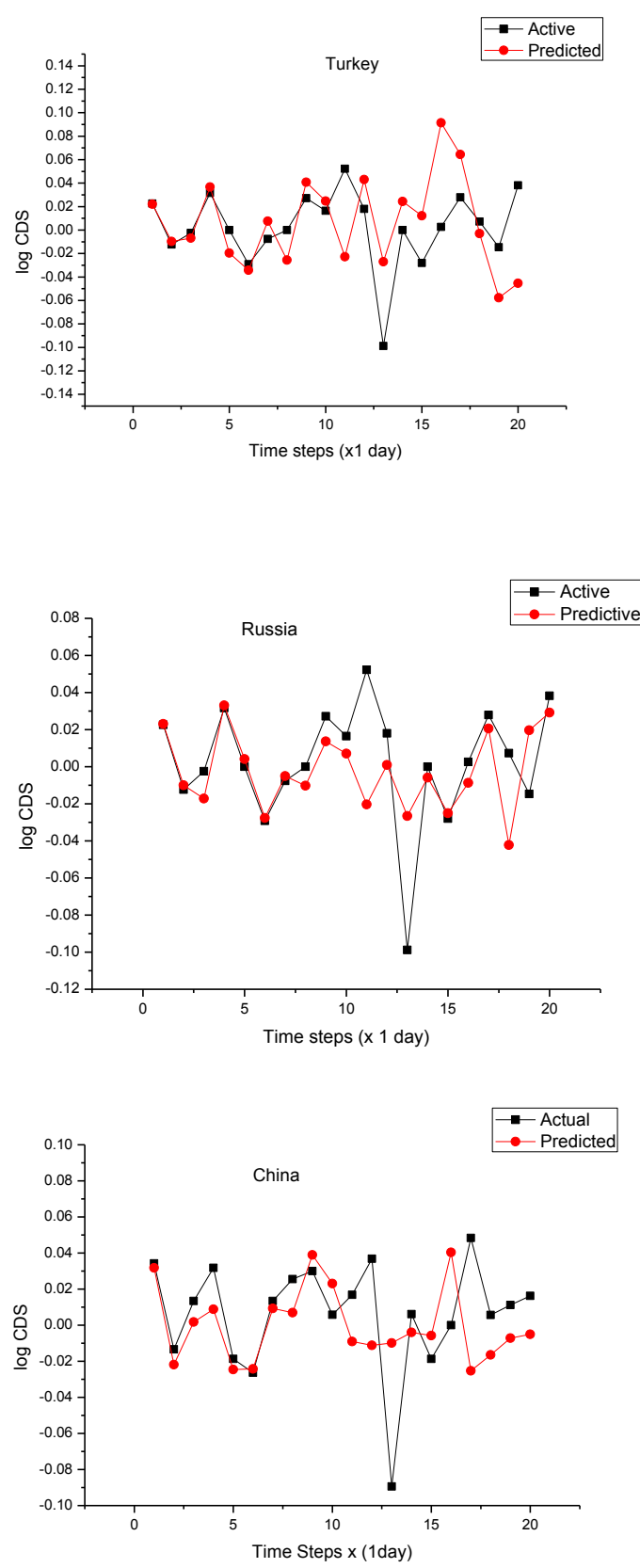

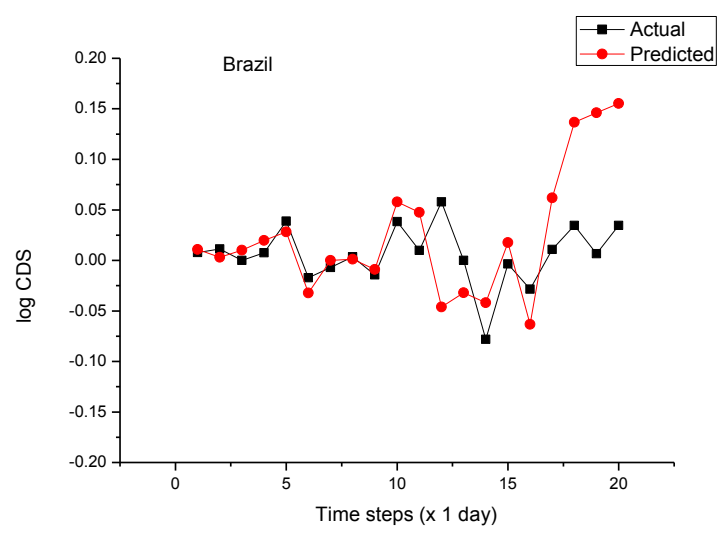

Fig.4 Actual and predicted out of sample predicted values

\section{Conclusion}

In this paper, we use a non linear analysis in combination with neural networks to predict the log CDS index for various countries. After estimating the minimum embedding dimension for each system, we point out that the systems are chaotic with high dimensionality. Based on the systems' strange attractors reconstruction we construct a backpropagation neural network with 2 hiddel layers. We achieved a reliable 10 time steps out of sample prediction. The empirical results can be used in economic prediction and trading purposes. Firstly, the emprical evidence can be used in practice to estimate the credit risk premiums of the countries. CDSs are used as risk premium priced by the markets. An efficient and correct prediction of CDS values will enable to the economist to have quantitative information on default risk in an economy. They can be used for country risk pricing, economic modelling and ratings. In the practical finance, the CDSs are traded in the markets. Thus, predicting future values of CDS has a potential earning opportunity for the market participants.

\section{References}

1. Medio, A. Chaotic Dynamics, Theory and Applications to Economics, Cambridge University Press, Cambridge, (1992).

2. Alexandre, H., Girerd-Potin, I. and Taramasco, O. "High frequency exchange rate forecasting by the nearest neighbour method", in Dunis, C. and Zhou, B. (Eds), Nonlinear Modelling of High Frequency Financial Time Series, Wiley \& Sons, New York, NY, (1998).

3. Finkenstadt, B. and Kuhbier, P. "Forecasting nonlinear economic time series: a simple test to accompany the nearest neighbour approach", Empirical Economics, Vol. 20, pp. 243-63, (1995).

4. M. Hanias, M., Curtis,P. and Thalassinos, J. "Prediction with Neural Networks: The Athens Stock Exchange Price Indicator" European Journal of Economics, Finance And Administrative Sciences - Issue 9,pp.,21-27,(2007)

5. Kantz, H. and Schreiber, T "Nonlinear Time Series Analysis", Cambridge University Press, Cambridge, MA.,(1997).

6. Takens, F. "Detecting strange attractors in turbulence", in Rand, D. and Young, L.-S. (Eds), Dynamical Systems and Turbulence, Warwick 1980, Lecture Notes in Mathematics, 898 Springer, Berlin, pp. 366-81,(1981).
7. Fraser, A.M. and Swinney, H.L. "Independent coordinates for strange attractors from mutual information", Physical Review, Vol. A No. 33, pp. 1134-45, (1986).

8. Abarbanel, H.D.I. “ Analysis of Observed Chaotic Data”, Springer, New York, NY.,(1996).

9. Kennel M.B., Brown,R. and Abarbanel,H.D.I., Phys. Rev. A 45 3403, (1992).

10. Cooper C., Chaos, Solitons Fractals 24, 157 ,2005)

11. Boudjema, G.and Cazelles,B. Chaos, Solitons Fractals 12, 2051 ,(2001).

12. Kugiumtzis,D., Lillekjendlie,B. and N. Christophersen, N.Model. Identif. Control 15, 205, (1994).

13. Wan,E.A. "Temporal Backpropagation: An Efficient Algorithm for Finite Impulse" Response Neural Networks, Proc. of the 1990 Connectionist Models Summer School, SanMateo, CA.,(1990).

14. Widrow.B. and Lehr ,M.,"30 Years of Adaptive Neural Networks: Perceptron, Madaline, and Backpropagation", IEEE Proceedings, vol. 78, No. 9, Sept. 1990, pp. 1415-1442, (1990). 